\title{
Les oasis du Monde, carrefour des civilisations et modèle fondamental de durabilité
}

\author{
Driss Fassi* \\ Comité national MAB du Maroc, Institut agronomique et vétérinaire Hassan II, Rabat, Maroc
}

\begin{abstract}
Résumé - Les oasis offrent un éclatant foisonnement de vie en plein désert chaud, là où la nature agressive développe justement une tyrannie absolue. Il est remarquable, de même, qu'elles aient quasiment monopolisé l'épanouissement des toutes premières grandes civilisations humaines, avec notamment les immenses oasis d'Egypte et de Mésopotamie. Les oasis du Moyen Orient ont été ainsi, pendant des millénaires, des centres de rayonnement universel, de qualité supérieure, et leur modèle a été adapté et généralisé de proche en proche au monde connu. Elles ont eu un caractère structurant pour l'ensemble de la démarche des civilisations humaines. Il suffit de rappeler qu' on y a mis au point pour toujours, les principaux cultivars de consommation de masse, la sélection majeure des animaux domestiques, les quartiers d'établissement sédentaire, et la gouvernance centralisée des nations. C'est, de toute évidence, le modèle fondamental de la durabilité. On n'en dira pas davantage, le sujet historique étant largement documenté. Le présent propos est surtout un questionnement autour d'exemples oasiens, actuellement remarquables, pour en tirer des enseignements de développement durable. C'est ainsi que, d'un continent à l'autre, on aura à consulter les Amériques arides, ainsi que l'ExtrêmeOrient chinois, les fastueuses métropoles d'Asie centrale, telles que Samarkand ou Tachkent, pour en revenir aux oasis recluses du grand Sahara africain, à la recherche d'une éventuelle authenticité. Ainsi outillé, on pourrait tenter, tout d'abord, d'opérer des choix utiles, dans la voie du développement adapté aux vastes régions oasiennes elles-mêmes. Mais bien au-delà, on pourrait se joindre aux efforts du Monde, et aller au-devant de cette transition écologique vers le développement durable, si recherchée. La zone oasienne, qui est aux avantpostes universels de la lutte contre la désertification, apparaît, en effet, comme un réel sélectionneur de terrain, des comportements censés mener vers un développement plus durable, pour l'humanité toute entière.
\end{abstract}

Mots clés : désertification / civilisation / développement / tradition / industrialisation

\begin{abstract}
The oases in the world, crossroads of civilizations and fundamental model of sustainability. The oases offer a dazzling abundance of life in hot deserts, where aggressive nature, precisely, develops its absolute tyranny. It is remarkable, also, that they have virtually monopolized the development of the first major human civilizations, with the huge oases of Egypt and Mesopotamia. The oases of the Middle East have been, for thousands of years, universal centers spreading a civilization of high quality, and their model has been adapted and generalized, gradually, to the known world. They had a structuring character for the entire process of human civilization. This model has developed the main cultivars of mass consumption, enabled the major selection of domestic animals, provided human sedentary establishment, and centralized governance of the nations. It is, obviously, the fundamental model of sustainability. We will not say more, the historical subject being widely documented. The present article looks at different examples of outstanding oases to learn lessons of sustainable development. To do so, we will consult, from one continent to the other, the arid Americas, as well as the Chinese Far East, the old lavish metropolises of Central Asia, such as the multifaceted Samarkand and Tashkent, going back to the recluses oases of the great African Sahara, looking for a possible authenticity. Thus equipped, we could try, first, to make useful choices, in the path of development suited to the vast oases regions themselves. However, beyond that, we could join in the efforts of the world, and go to the front of this ecological transition towards sustainable development, so sought after. Indeed, the oasis area, which is the universal front line of the struggle against desertification, appears as a real field breeder, mastering behaviors that are supposed to lead to a more sustainable development for all humanity.
\end{abstract}

Keywords: desertification / civilization / development / tradition / industrialization

\footnotetext{
$\overline{{ }^{*} \text { Auteur de correspondance }}$ : idrissfassi@yahoo.fr
} 


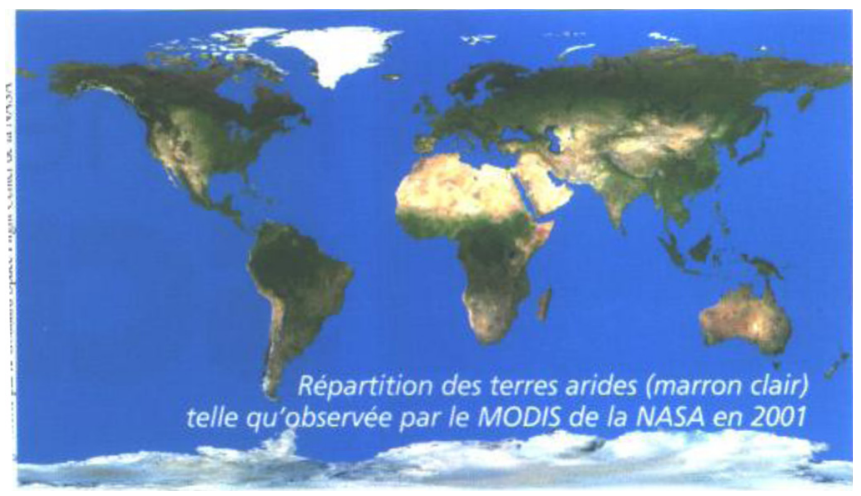

Fig. 1. La zone saharienne, désespérément dépouillée, mutile le couvert biotique de la Terre.

Fig. 1. The Sahara, an area without vegetation cover.

Les oasis sont des entités d'exception, qui peuvent foisonner de vie à leur échelle très ponctuelle, au sein d'immenses déserts biologiques, sur la base des éléments premiers des écosystèmes. C'est pourquoi la moindre erreur d'aménagement peut être fatale. La sanction frappe alors au plus serré, et la leçon est immédiate et explicite, car le désert, qui en est le contexte zonal ordinaire, revient et reprend ses droits. Ainsi, les oasis s'affirment comme étalon de l'espace habitable tout entier, celui des équilibres fondamentaux, ne permettant de consommer que les ressources renouvelables, et selon les rythmes de leur régénération.

Elles sont notamment utilisées pour apporter des éléments de réponse aux questions majeures qui se posent à l'environnement.

«Les changements climatiques» peuvent considérer les oasis comme matériel frontal hypersensible, posté en avant $\mathrm{du}$ couvert biotique, et prêt à enregistrer les moindres modifications.

La notion de "désertification», qui peut en être un indicateur principal, a beaucoup évolué dans l'opinion. Il y a juste 50 ans, on proclamait encore la toute-puissance de la technologie, apte à relever les défis de tous les milieux. Aujourd'hui, on estime universellement qu'il n'y a de salut que dans le respect du développement durable, en confirmation de la règle d'or qui a pérennisé la civilisation oasienne depuis l'Égypte protohistorique.

Le « développement durable», qui serait la clé même de pérennité de l'humanité, devrait donc s'inspirer du modèle oasien, en tant qu'étalon pratique de base. L'histoire globale des civilisations semble montrer qu'il s'agit d'une référence perpétuelle, qui a souvent su se rendre utile (Ibn Khaldoun, 1377).

Le phénomène oasien, ainsi concept central, peut être évalué géographiquement, à travers l'étendue des déserts chauds où il s'inscrit. Ces déserts, véritable rupture biotique (Fig. 1), coïncident parfaitement avec la ceinture anticyclonique subtropicale permanente, qui interdit systématiquement la pénétration des masses d'air humides, et prête une allure magique aux rares apparitions oasiennes.

L'emplacement précis des déserts, et la définition de leur taille, sont en rapport avec des données géographiques particulières. Il s'agit normalement de basses latitudes, plus rarement moyennes, et qui n'affectent que les façades occidentales des continents. Le modèle accompli en est le grand désert africain et ses prolongements asiatiques décalés vers le nord, donnant les ensembles arides les plus vastes et les plus diversifiés, car ils rajoutent à leur rente de position, celle de la massivité des trois vieux continents coalescents. En bon représentant de l'espace traditionnel du monde oasien, il est censé nous fournir les spécimens les plus intéressants, à la recherche du développement durable en milieu aride.

D'autres facteurs commandent l'apparition des oasis: particulièrement l'affleurement des nappes souterraines, et l'existence de cours d'eau allogènes. Les réserves hydriques fossiles, accumulées lors de paléoclimats humides, en constituent le fond constant, alors que les éventuelles montagnes bordières leur fournissent les eaux renouvelables, en proportion de leurs étages bioclimatiques et de leurs pentes.

\section{Des systèmes oasiens hors-normes, placés aux limites du possible}

L'aménagement des «zones arides américaines», de par l'ingéniosité investie et les moyens consentis, obligent à de la déférence. L'industrie aéronautique, et globalement militaire, les camps d'entraînement et gigantesques dépôts de matériel roulant de grande sophistication... sont au firmament du genre, adaptés qu'ils sont aux types de temps extrêmement secs des déserts, Mojave et Sonora, clairs et non corrosifs, et à la bonne tenue des sols. D'autres secteurs tout aussi stratégiques, tels que la forte concentration d'observatoires, ainsi que la légendaire industrie cinématographique, ont profité des mêmes conditions subtropicales (Barnes et Pendleton, 1979). Cependant, l'immense urbanisation, proprement explosive, a fait que Los Angeles, deuxième plus grande ville des États-Unis d'Amérique (EUA), a multiplié la population de la cité par 1800 en 150 ans, pendant que son aire métropolitaine s'était étalée sur une centaine de kilomètres, pour approcher les 20 millions d'habitants, le tout sous un apport pluvial qui ne dépasse guère les $300 \mathrm{~mm} / \mathrm{an}$. Las Vegas, célèbre capitale mondiale des jeux de hasard, est aussi la troisième destination américaine pour les congrès et les réunions d'affaire, et un haut lieu touristique incomparable. Elle tente de compenser le peu d'eau qu'elle reçoit du ciel $(<100 \mathrm{~mm})$, les chaleurs accablantes et la faible humidité de l'air $(<10 \%)$, par un système monumental de jeux d'eau, qu'on a qualifié de « délire aquatique». Pour justifier une telle débauche de ressources précieuses, la Commission de l'eau de la ville a pu dire que «chaque million de litres d'eau décorative génère 30 millions de dollars de revenus pour le Nevada ». C'est apparemment avec le même esprit que tout le pays aride américain a été géré, alors que, du fait des rigueurs climatiques, il lui faut trois fois plus d'eau pour générer le même confort que sur la côte Est plus humide, qui consomme elle-même le décuple de la consommation en eau des pays du Maghreb.

À ce rythme, l'Amérique engloutirait volontiers les réserves du monde. The North American Water and Power Alliance (NAWAPA) est un excellent révélateur d'intention, donnant l'échelle géographique et technologique que seule une nation de ce gabarit est capable de développer. Le projet se propose de détourner de gros cours d'eau de l'Alaska et du Canada, au profit du Canada, des EUA et du 


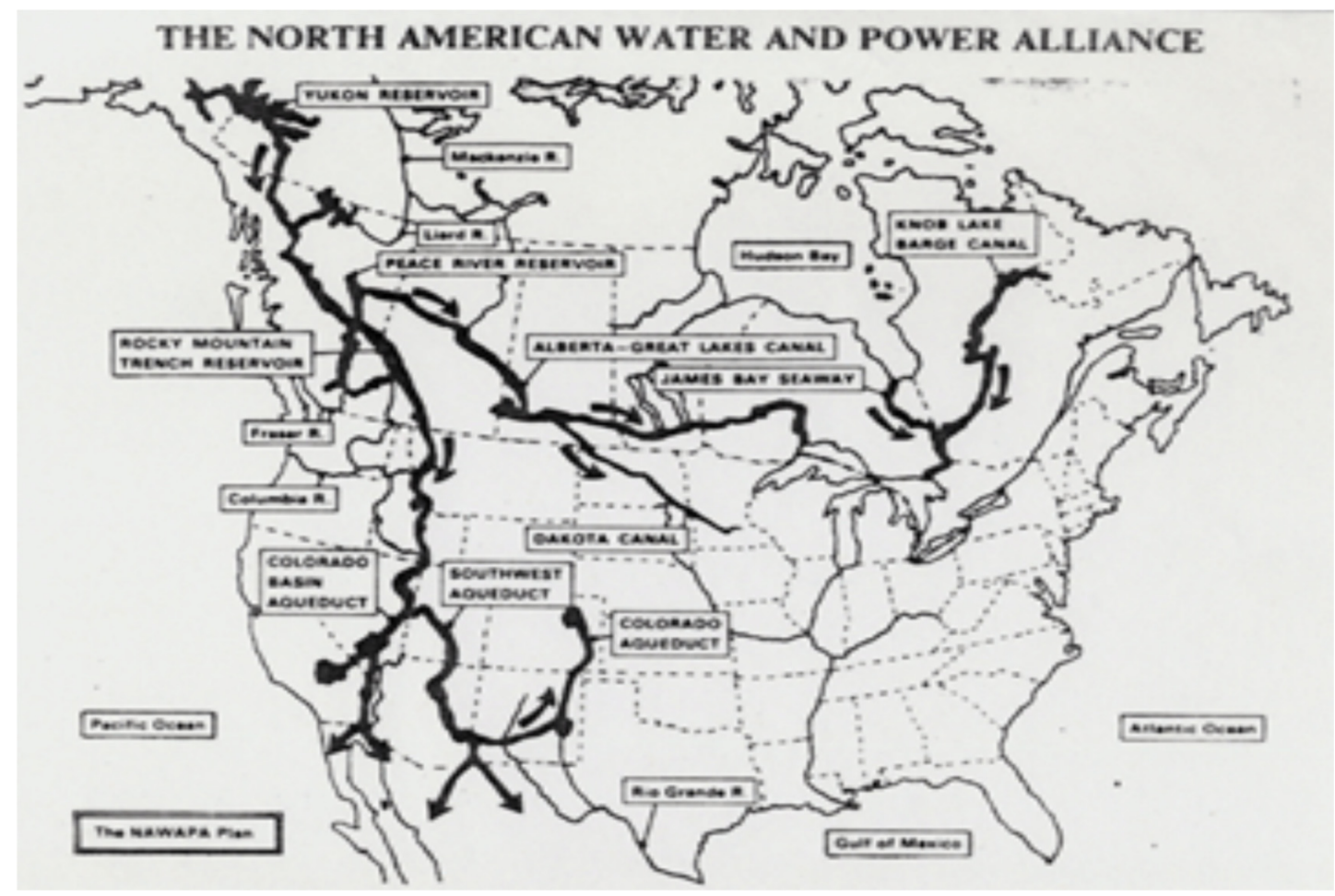

Fig. 2. Réseau de captures hydrographiques, selon le projet NAWAPA.

Fig. 2. The NAWAPA, a proposed continental water management scheme.

Mexique (Fig. 2), dont en bonne partie le sud-ouest aride des États-Unis. Pour donner l'échelle du chantier, le Mexique, qui n'était censé recevoir que moins de $3 \%$ du volume général de l'eau à mobiliser, parviendrait quand même, grâce à cet éventuel apport, à tripler la totalité de ses terres irriguées. Les cours d'eau en question, qui se déversent en bonne part dans l'Océan Arctique, déjà extrêmement fragilisé par l'action humaine, seraient déviés dans le sens opposé de leur cheminement naturel, pour se perdre dans les gouffres incommensurables de la consommation américaine.

Le projet est encore pour le moment politiquement non réalisable, mais tout à fait significatif de la tendance. Un autre projet démesuré, pourrait opérer à partir des antipodes, avec le remorquage envisagé des icebergs de l'Océan glacial Antarctique. On serait avec les deux, à l'affût d'une batterie de profondes mutilations, éventuellement en interférence avec les grandes cellules anticycloniques des pôles, autre moteur permanent majeur de la circulation atmosphérique générale, et facteur possible des changements climatiques.

Il semble assez plausible que la philosophie de gouvernance des terres arides du sud-ouest américain mette la promesse de l'american dream bien au-dessus du souci de développement durable. Mais, la bonne nouvelle consiste justement à envisager un tel élan solidaire, quels qu'en soient les objectifs. C'est de toute évidence à une échelle sans précédent que des pays performants envisagent de monter ensemble un complexe de projets de développement, et qu'ils semblent prêts à mettre à contribution territoires, génie et moyens. Il suffirait pourtant de rester en phase avec les efforts de développement déjà consentis, parfaitement adaptés aux sites, et dont la région est passée championne, du genre stations d'expérimentations aérospatiales et de stockage du matériel lourd des puissantes industries de pointe, ou de l'adoption intensive des énergies renouvelables. Une stratégie nationale, du même ordre que celle qui a amené la maîtrise du Mississipi par exemple, pourrait se fixer comme objectifs de réduire les déserts et d'orienter les établissements humains tentaculaires vers des solutions d'éco-urbanisme. À cette nouvelle échelle, sans aller à la coalition supranationale et sans cesser d'être efficace, le projet coûterait dix fois moins cher que ne pourrait l'être la NAWAPA, et s'installerait dans la durée, pour corriger des dichotomies spatiales très préjudiciables.

Cependant, on pourrait comprendre que la nation ne soit pas encore en état d'être mobilisée pour une cause environnementale universelle de ce niveau. Los Angeles, qui en est une vitrine des plus fiables, compte près de 150 nationalités, et parle peut-être 230 langues. Il n'y a sûrement pas meilleure façon de mettre tout ce monde en harmonie que de montrer, par tous les moyens, que «le rêve continue».

À l'autre bout du monde, l'autre superpuissance est dûment en train d'élaborer « la grande muraille verte de Chine». Elle y met tous les ingrédients pour en faire le processus de protection de la nature le plus massif de tous les temps. Sur une envergure de près de $5000 \mathrm{~km}$, et à coups de milliards de dollars, c'est un projet continental, d'un siècle de mise en train, qui se propose de stopper le Désert de Gobi, dont la progression enterre chaque année plus de $3000 \mathrm{~km}^{2}$ de prairies en Chine du Nord. Le pays plante plus d'arbres que tout le reste du monde, et encadre particulièrement dans cet effort les paysans proches des milieux dégradés. Il stabilise les champs de dunes par des plantations adaptées, déclare que les plantations à croissance rapide absorbent davantage de gaz à effets de serre, tente 


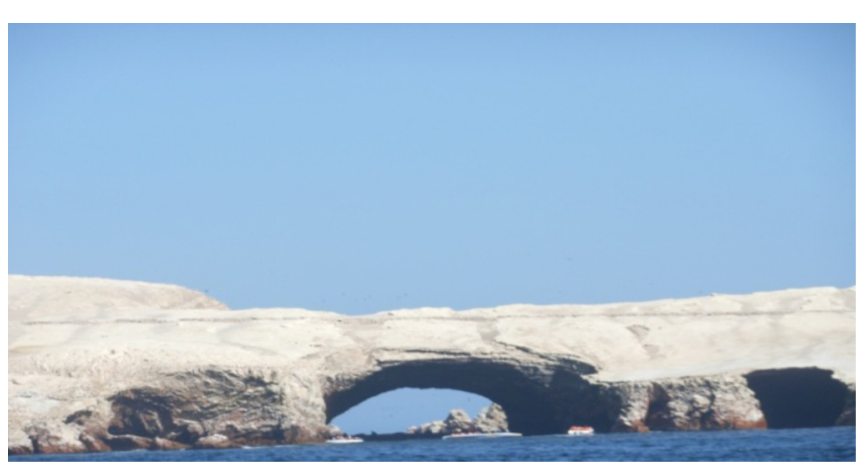

Fig. 3. Formes littorales désertiques au Pérou, chargées de guano. Site grouillant de biodiversité marine.

Fig. 3. Coastal desert in Peru with guano deposits attesting the richness of local marine biodiversity.

l'enclenchement des pédogenèses, et est censé protéger, dans le même élan, le Japon et les Corées. Il y a certes également de nombreuses marques d'échec, mais le projet dispose encore de plus de 60 ans pour apprendre et corriger.

À côté de ces deux pôles, que le progrès technologique ne cesse de rapprocher, il se trouve des expériences hors-normes, qui tentent de prêter aux déserts un rôle inédit.

«En Amérique du Sud», le désert est le plus souvent un espace marginal, qui ne possède pas de réelle tradition oasienne et ne semble pas avoir de leçon à dispenser, sauf peutêtre l'expérience du Pérou. C'est d'abord la configuration du pays qui, contre toute attente, met en avant son désert, bande littorale ainsi placée au contact du monde des affaires. Elle occulte tout autant le cordon andin, montagne périlleuse et pays de vestiges inca, dédié au tourisme, et la forêt amazonienne très sous-équipée et extrêmement enclavée. L'Atacama, au sud de Lima, est même l'un des déserts les plus absolus qui soient. L'aire métropolitaine de la capitale concentre près $\mathrm{du}$ tiers de la population nationale, et commande la majeure partie des activités du littoral. L'activité minière et les zones industrielles interfèrent avec les périmètres irrigués, les côtes à guano en perte de vitesse, relayés par le tourisme qui exploite le pittoresque des côtes, et surtout la diversité biologique qui en anime notamment les baies et îlots proches, souvent organisés en réserves naturelles (Fig. 3). Celles-ci grouillent d'arthropodes spécialisés, d'oiseaux très divers, et de mammifères marins, et laissent même voir ces mystérieux géoglyphes, qu'entourent les légendes. À côté des aspects dominants des grandes villes occidentales, des emprunts andalous (Fig. 4) apparaissent dans certains quartiers de la ville, et même des équipements sahariens, notamment en matière d'exhaure et d'irrigation, tels que les canaux souterrains du type khettara (foggara dans le Maghreb central, qanat en Iran).

\section{Les oasis d'Asie centrale développeraient des prototypes d'évolution, de tradition en modernité}

Les oasis d'Asie centrale, depuis la Mer Caspienne jusqu'en Chine, jalonnent des déserts de moyennes latitudes, à hiver froid. Le froid devient plus intense vers l'est, pour finir

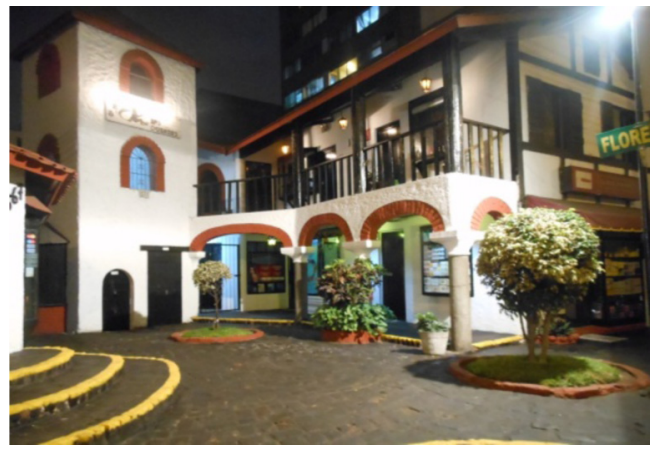

Fig. 4. Type de résidences andalouses (quartier Miraflores de Lima). Fig. 4. Moorish Andalusian mansions in Lima (Miraflores).

avec le désert de Gobi, dans un bassin hyperaride de plus d'un million de kilomètres carrés, aux précipitations insignifiantes, et aux amplitudes thermiques saisonnières records, atteignant près de $80^{\circ} \mathrm{C}$. Les étés sont torrides, et les hivers font de la région le désert le plus froid au monde, après l'Antarctique. Ce sont, avec le Xinjiang chinois, les maillons extrêmes de ce qu'était la «route de la soie», voie perpétuelle de grand négoce qui reliait la Chine orientale à la Syrie. Les maillons réputés s'en trouvaient en Asie centrale, avec ces fastueuses cités aux noms magiques, que sont Samarkand, Boukhara ou Tachkent. Espace oasien particulier, avec son étirement transcontinental et son expérience millénaire, il semble réunir les ingrédients pour faire école en matière de développement en milieu aride.

Tout aurait commencé par l'homogénéisation ethnique et linguistique, grâce aux migrations issues des beaux massifs humides, englacés et forestiers de l'Altaï, joignant la Chine à la Sibérie. La montagne serait la patrie d'origine des communautés turques, qui ont sillonné le continent jusqu'aux confins occidentaux de l'Asie, pour continuer en Europe. D'autres éléments humains ont fixé le caractère confessionnel régional dominant, qui s'est perpétué, la sophistication apportée aux réseaux d'irrigation, et l'esprit du négoce ouvert sur le monde, en rapport avec l'intégration au califat omeyyade. Le califat abbasside a rajouté à la structuration de l'administration, et à la généralisation des monnaies de ces dynasties (viii ${ }^{\mathrm{e}}$-xiii ${ }^{\mathrm{e}}$ siècles), le resplendissement de grands noms dans tous les domaines des connaissances (Fig. 5).

Tout aussi marquantes ont été les influences iraniennes, et surtout l'importante période de gouvernement mongol, dont il faut absolument distinguer la période faste du second empire, âge d'or inauguré par Timour Lenk (Tamerlan, xiv ${ }^{\mathrm{e}}$ siècle), et ses descendants, les émirs timourides, qui ont fait de Samarkand la capitale de l'empire continental le plus vaste de tous les temps (Fig. 6).

Enfin, les nations de l'Asie centrale en sont arrivées à partager, pour près d'un siècle, le sort extraordinaire de la Russie, à travers les événements de fin d'empire, puis de l'Union soviétique, jusqu'à la chute du système au début des années 1990 du siècle dernier. C'est pour ce monde oasien précis, un tournant décisif qui s'exerce, en utilisant les moyens d'une superpuissance, à tester les ressorts de durabilité de toutes les activités de développement en milieu aride.

À l'avènement du pouvoir russe, l'Asie centrale offrait l'image de petites régions climatiquement sèches, au passé certes assez prestigieux, mais qui s'étaient retrouvées dans une 


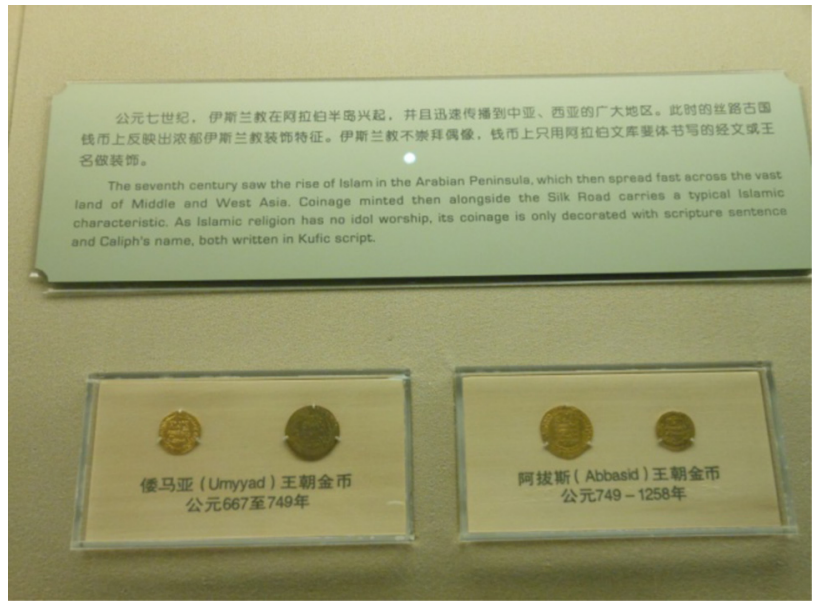

Fig. 5. Phases d'épanouissement du négoce sur la Route de la soie, les monnaies des dynasties omeyyade et abbasside (viii ${ }^{\mathrm{e}}$-xiii ${ }^{\mathrm{e}}$ siècles$)$, battues et en cours tout au long du parcours, font foi (collection du musée de Shanghai).

Fig. 5. Umayyad and Abbasid money (8th-13th centuries) along the Silk Road testify from the important trading activity (Shanghai Museum).

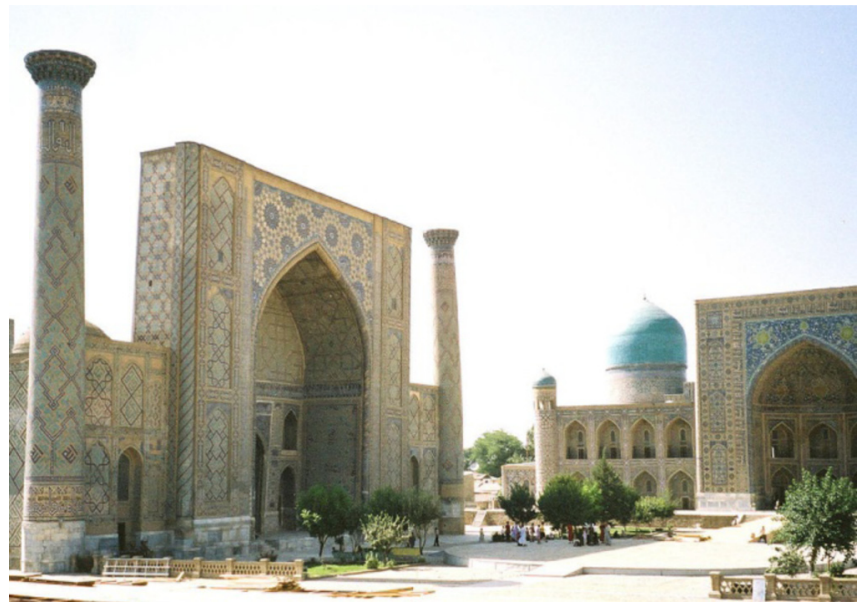

Fig. 6. Registan, pôle de vie religieuse, commerciale et artisanale de Samarkand. Madrassa, centres de rayonnement scientifique et culturel ( $\mathrm{xv}^{\mathrm{e}}-\mathrm{xvii}{ }^{\mathrm{e}}$ siècles).

Fig. 6. Samarkand: religious, commercial and artisanal center with an important scientific and cultural influence (15th-17th centuries).

relative léthargie socio-économique, rappelant ainsi certains des pays du Maghreb et du Moyen Orient. Elles vont assez brutalement être projetées dans le giron d'un grand pays qui ne visait rien de moins que la prééminence économique et militaire universelle.

Parmi les immensités du Kazakhstan on a trouvé de l'espace pour les essais nucléaires, et pour le cosmodrome de Baïkonour, base renommée de lancement des fusées soviétiques.

Plus nettement axé sur le développement régional, c'est de nouveau le nord du Kazakhstan qui est investi pour défricher, au milieu des années 1950, jusqu'à 40 millions d'hectares de steppes (Lahmar, 1997), mobiliser plus de 300000 jeunes au

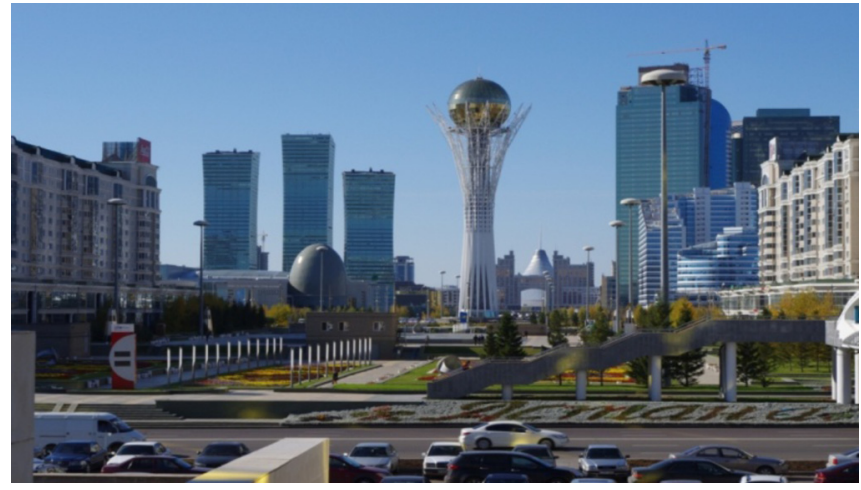

Fig. 7. Astana, capitale politique et économique du Kazakhstan.

Fig. 7. Astana, political and economic capital city of Kazakhstan.

nom du socialisme scientifique, ouvrir un immense front pionnier, et produire des céréales à perte de vue pour nourrir les masses à l'échelle nationale. L'action s'est exercée sur la vaste «steppe kazakhe», essentiellement au nord du $50^{\mathrm{e}}$ parallèle, avec des précipitations pluvio-nivales de l'ordre de $300 \mathrm{~mm}$, et des amplitudes thermiques considérables, comme il sévit largement dans ces plaines profondément continentales. La ville qui a servi pour commander et équiper cette opération culte, dite des terres vierges, a été baptisée Astana (capitale en kazakh), et donc toute désignée pour devenir la capitale actuelle de la république (Fig. 7).

Avec les années 1960, les Soviétiques s'attachent à établir les grands chantiers sur les terres arides du Sud, pour faire produire du coton aux déserts du Turkestan, à une échelle de rentabilité sans précédent. Le projet consistait à mettre en synergie les immenses glaciers du Pamir, juchés jusqu'à plus de $7000 \mathrm{~m}$ d'altitude, et le fabuleux réseau hydrographique de l'Amou-Daria et du Syr-Daria, généreusement alimenté par la fonte estivale des neiges, pour l'amener à féconder près de 7 millions d'hectares dans les terres brûlées du bas pays touranien. Tour de force qui fait qu'actuellement encore, la république d'Ouzbékistan par exemple, qui est aride à $90 \%$ de son territoire, est le $4^{\mathrm{e}}$ producteur et $2^{\mathrm{e}}$ exportateur mondial de coton...

Globalement, le projet des «terres vierges » s'est soldé par une baisse généralisée des rendements, due à la troncature massive des horizons arables, menacés d'un dust bowl comme dans le Midwest américain. Mais ce n'est encore rien par rapport aux bouleversements induits dans les terres arides du Sud du Kazakhstan, en Ouzbékistan et au Turkménistan, où les équilibres naturels ont été entièrement bouleversés. Le réseau hydrographique, utilisé pour la monoculture cotonnière, étant de nature endoréique, il était parvenu à s'organiser autour de la mer intérieure d'Aral, pour s'y jeter par des formations deltaïques, notamment très riches en produits dissous. La grande agriculture moderne, qui prélève de 60 à $90 \%$ des débits naturels, a mutilé le réseau, et la mer qui couvrait à l'origine près de $70000 \mathrm{~km}^{2}$, a été réduite de moitié au tournant du siècle, et devrait normalement céder complètement la place à un désert d'Aral dans moins d'une dizaine d'années (Fig. 8). De plus, les remaniements éoliens, devenus beaucoup plus corrosifs, sont en train de causer une réelle endémie de maladies respiratoires et de la peau, voire d'affections cancéreuses. 


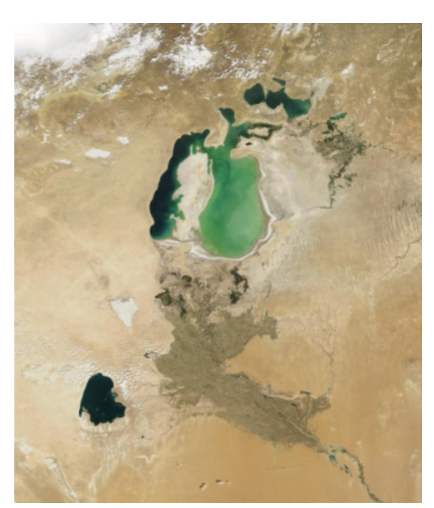

Fig. 8. La Mer d'Aral, et la menace de disparition dans les sables du bas pays de l'Asie centrale.

Fig. 8. The Aral Sea, disappearing in the sands of Central Asia.

Maintenant, il y a matière à réflexion concernant «l'industrialisation des zones arides», et c'est certainement pour la cause, l'un des meilleurs observatoires possibles. En effet, lors de la Seconde Guerre Mondiale, l'URSS a utilisé les républiques d'Asie centrale comme terrain de repli pour les populations européennes fragilisées, et surtout comme destination d'évacuation pour les industries, notamment stratégiques et lourdes. Il semble bien que, globalement, on n'ait pas à s'en plaindre, et que des cités en milieu aride, qui s'étaient ainsi convenablement industrialisées, telles que Samarkand, ou même plus tard des régions comme au Kazakhstan, se portent beaucoup mieux que des pays montagnards de la région qui n'ont pas eu cette chance, et qui sont alors restés nettement sous-équipés.

\section{Les zones hyperarides centrales, avec le Sahara au cœur des traditions oasiennes}

C'est bien au centre de l'espace habité global que s'étale paradoxalement le Sahara, le plus grand désert chaud du monde. Depuis la fin des glaciations (Fassi, 1999), la civilisation sédentaire globale est assurément née, et les domestications utiles se sont élaborées au Moyen Orient, puis se sont concentrées dans les grandes vallées et massifs montagneux de l'actuel Sahara, au fur et à mesure des aridifications climatiques (Guilaine, 2000). Il en est résulté cet immense hiatus biotique, essentiellement africain, augmenté $\mathrm{du}$ désert arabique dans son prolongement oriental, à concurrence de près de 12 millions de $\mathrm{km}^{2}$. Les centres de prédilection de la décision et de l'innovation n'ont pas cessé d'être des oasis, des milliers d'années durant. Puis, leur importance s'est progressivement réduite, pour ne plus constituer que des maillons de communication entre les grandes nations commerçantes, jusqu'au début des temps modernes où les colonisations ont changé les mécanismes et les flux internationaux; les rapports à travers le Sahara sont alors apparus comme totalement obsolètes. Aujourd'hui, il n'y a plus que ces réseaux d'oasis, tels que le Draa, le Tafilalt, la Saoura et les Touat, jusqu'à Ghadamès, Ghat et Djanet, qui tiennent leurs eaux essentiellement des Atlas, et s'insinuent au plus secret des solitudes sahariennes, en direction de leurs massifs centraux, qui peuvent prétendre être encore déposi- taires de sites et de technologies oasiens, doués d'une certaine authenticité (Fig. 9).

On peut encore observer des terroirs oasiens stratifiés (Fig. 10), avec des palmiers dattiers, couvrant des fruitiers méditerranéens, au-dessus de petits lots ciselés selon des normes d'une belle sophistication, où alternent cultures vivrières et fourragères, avec des spéculations traditionnelles du type henné, safran, gombo ou roses. Plus précieux encore, mais en perdition rapide, est cette capacité de l'aride à développer des «variétés endémiques » dans tous ces secteurs, et tout autant parmi le bétail local, le tout présentant des facultés d'adaptation exemplaires aux rigueurs du milieu, et des propriétés médicinales et organoleptiques d'exception. Une telle agro-diversité du terroir aurait été acquise par une mise à l'épreuve plusieurs fois millénaire, entre une chaîne atlasique élevée et continue, qui garantit l'isolation au nord, et un souffle puissant d'air sec épurateur, émanant directement du cœur du Sahara, pour concentrer les éléments nutritifs et actifs, utiles.

L'aménagement oasien consisterait en la recherche d'«alliés de durabilité», naturels ou technologiques éprouvés, vis-à-vis des conditions extrêmes du milieu saharien. Ils garantissent eau, sols et diverses immunités contre les agressions des conditions extrêmes. Dès qu'un produit biotique, végétal ou même animal, parvient à devenir un facteur structurant de l'oasis, il acquiert quasi forcément les qualités de l'endémisme, du fait des rigueurs du milieu, qui apparaissent comme un «raffinage» édaphique, impliquant la robustesse des organismes biotiques, ainsi puissamment aguerris contre les conditions extrêmes.

On s'en est rendu compte avec les oasis de l'Asie centrale, où les formes oasiennes de premier ordre appartiennent à des réseaux longilignes, disposés le long de cours d'eau allogènes. Elles se rapportent aux ensembles de modèles nilotiques, ou mésopotamiens, références primordiales, qui sont des dons de la montagne, à quelque distance qu'elle soit. Au Maghreb, les réseaux d'oasis ramènent des Atlas eaux superficielles, forcément renouvelables, et particules terrigènes, car il semble bien que les milieux qui reçoivent des précipitations moyennes annuelles inférieures à $200 \mathrm{~mm}$ soient incapables d'assurer leurs propres pédogenèses de manière performante (Unesco, 2000). Afin de maîtriser l'irrigation, des séries de petits barrages de fortune, au fil de l'eau, en assurent la distribution, et sont remarquablement fusibles à la moindre crue, permettant ainsi l'extension des périmètres submersibles, en dépassement des limites ordinaires de l'endoréisme de chacun des flux qui porte la vie au désert, et repousse le front de désertification. Ces barrages, légers et fonctionnels, suffisent à piéger un maximum d'alluvions grossières au droit des talwegs, pendant que la submersion féconde les parcelles par les argiles les plus fines, et donc les plus fertiles. C'est ainsi que de vastes espaces, dans les dépressions arides placées en interfluves (maader), fournissent une production agricole traditionnelle d'appoint, grâce aux épandages de crue, dont la menace potentielle est tout autant déjouée, que très utilement mise à contribution.

Les autres alliés de durabilité deviennent plus technologiques, sans cesser d'être conformes à la logique de la nature et de la civilisation. Les systèmes d'irrigation et abreuvement sont complétés par les khettara, procédé le mieux adapté aux conditions sahariennes proches des piémonts. Il s'agit de 


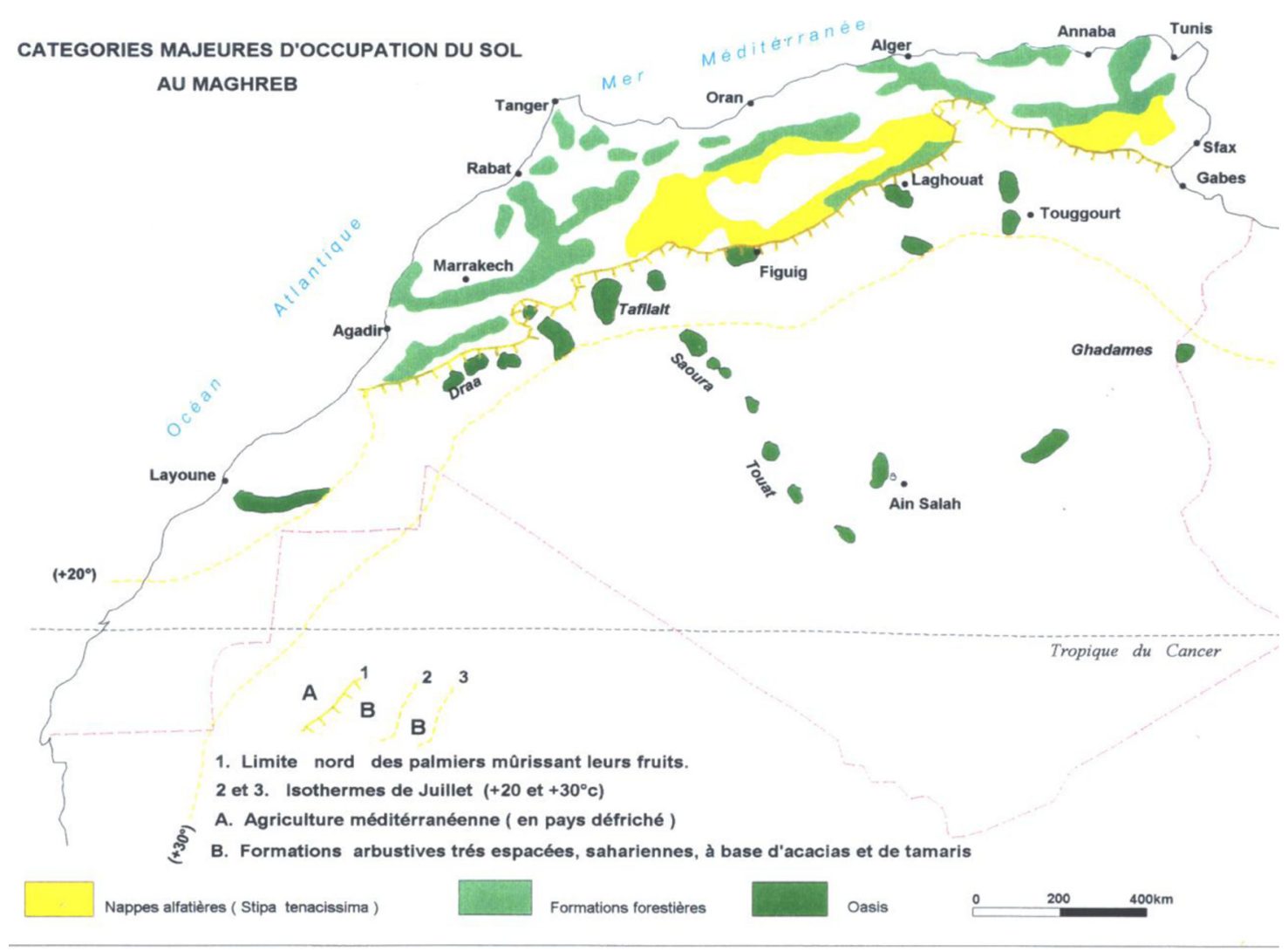

Fig. 9. La distribution des oasis du Maghreb, (Fassi, 2001), prend la forme de chapelets de globules foncés, qui soulignent les Atlas (Haut et Saharien), et s'enfoncent vers le sud-est pour rejoindre le massif du Hoggar.

Fig. 9. Oases distribution in Maghreb (Fassi, 2001): a string of dark green areas from the Atlas Mountains to the Hoggar Mountains.

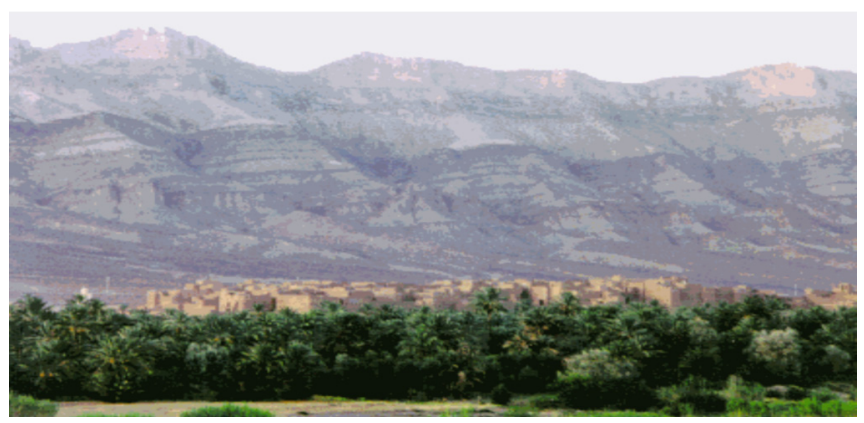

Fig. 10. L'agro-diversité oasienne stratifiée est commandée par l'habitat saharien de type ksar.

Fig. 10. Ksar, Sahara fortified village inside oasis vegetation.

travaux lourds qui exploitent les nappes phréatiques d'amont, et en orientent l'écoulement calibré par des canaux souterrains, vers des terroirs oasiens préétablis. De véritables ingénieurs traditionnels en assurent le fonctionnement. Au Maroc, on connaît par leur nom des communautés expertes, censées en fournir les cadres (celles du Dadess), et des traditions de travaux collectifs (touiza) pour en réaliser le creusement et l'entretien.

Un train de dispositions teintées de sacralité, et de techniques localement maîtrisées, accompagne toutes les opérations d'aménagement des oasis, jusqu'aux structures d'aboutissement représentées par l'habitat, appelées selon les

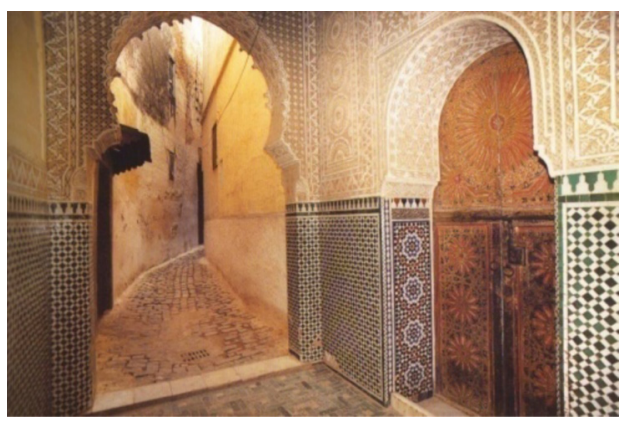

Fig. 11. Dans le ksar comme en médina (ville, ici, la médina de Meknès), l'habitat comme les systèmes d'irrigation sont des structures adaptées, savamment construites, et dûment collectives.

Fig. 11. In the Meknès Medina, houses and irrigation systems are adapted collective structures.

cas casbah (citadelle) ou ksar (forteresse). Le ksar, qui en est la formule collective accomplie, est normalement une unité autosuffisante et fermée, qui multiplie les formes d'adaptation par l'usage privilégié de l'architecture en terre, le choix des sites et des expositions, en évitant l'accaparement des terroirs agricoles, et en recherchant l'utilisation limitée des ressources par le recours systématique au service collectif (Fig. 11 et 12). Encore faut-il préciser que le ksar, en tant que quartier multifonctionnel et fortifié, est déjà une formule de repli cloisonné, défensive et donc relativement tardive de l'habitat 


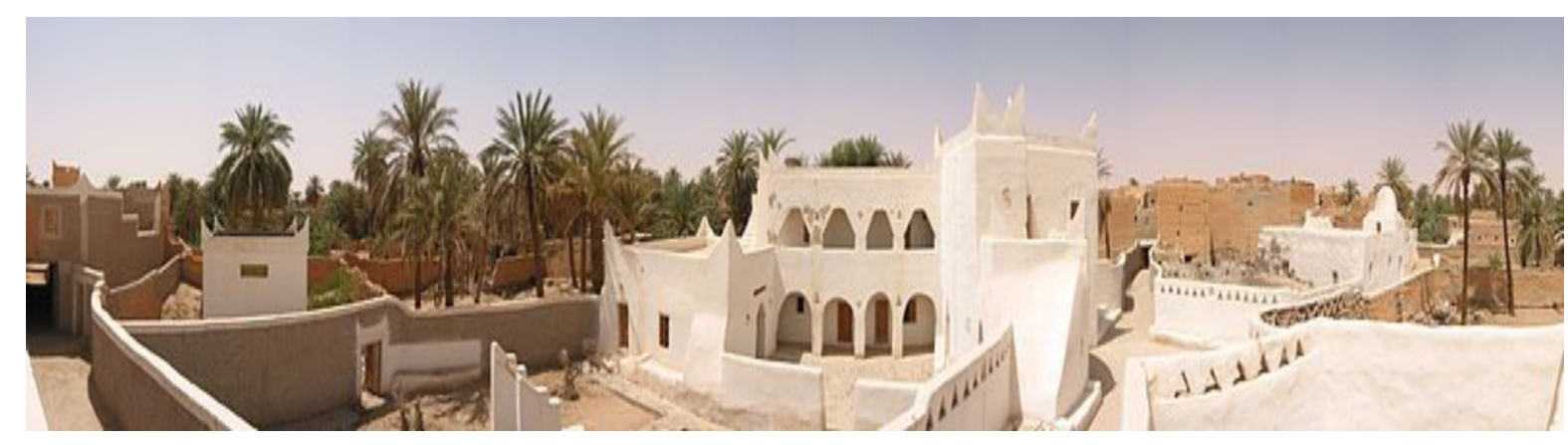

Fig. 12. Noyau originel de Ghadamès (Libye): structure citadine saharienne, entièrement construite, avec deux étages d'espaces vécus, strictement spécialisés.

Fig. 12. The Ghadames oasis (Libya): a Sahara city with two-storey houses.

oasien. L'habitat originel, comme illustré par la prestigieuse ancienne Sijilmassa (Miller, s.d.), était plutôt bien articulé sur son environnement, en symbiose avec la conformation hydromorphologique des sites fluviatiles ou des grands canaux d'irrigation. En tout cas, il s'agit d'une structure totalement construite, où transparaissent clairement les fonctionnalités socio-économiques et environnementales caractéristiques des médinas.

Le $\mathrm{xx}^{\mathrm{e}}$ siècle qui a imposé, à tous les niveaux, des modèles supposés universels, s'est inscrit en rupture dans l'évolution des oasis, par l'implantation de quartiers d'habitat moderne, l'éclatement des ksar, la construction de grands barrages, la multiplication des pompages d'eau dans le désordre et l'établissement de plantations en surnombre à partir des nappes souterraines. Au Maroc, les ksar partent à l'abandon, et sont vidés à plus des trois quarts. Certaines casbah ont été transformées en résidences touristiques. En Égypte, même dans les oasis occidentales, en dehors du grand axe du Nil, les ksar ont été désertés jusqu'au dernier, restaurés, et sont visités comme des musées.

En somme, même si l'évolution en semble irréversible, de nombreuses composantes de l'oasis traditionnelle, autant pour l'aménagement de l'espace, l'agro-diversité, et les modes d'irrigation, que pour l'esprit de l'habitat et l'expression culturelle, peuvent continuer à faire école.

En guise de conclusion, il ressort de l'expérience oasienne de par le monde, que le problème majeur concerne avant tout les choix d' "aménagement agricole». Dans la généralité des cas, l'agriculture accapare des proportions d'eau excessives, variant de 75 à $95 \%$ des disponibilités. En comparaison, les oasis sont une référence de gestion optimale de l'irrigation. Des perspectives d'amélioration existent certainement pour accroître la performance, et diminuer les pénibilités. L'important est de se garder de céder à l'escalade spéculative, basée sur les monocultures de masse, quelles qu'elles soient. L'expérience soviétique est, certainement de ce point de vue, le travers majeur à éviter. Par contre, les possibilités sont illimitées pour engager des produits du terroir, toujours d'une grande finesse (Bouaziz, 2000), dès l'instant qu'on a les moyens et l'autorité d'en faire la meilleure valorisation possible, et d'en contrôler fermement la commercialisation.

«L'industrialisation » en zones arides a toujours mauvaise presse, car ses nuisances sont concentrées, d'autant qu'en milieu sec, les dilutions sont difficiles, et que les zones en dépression qui y sont très fréquentes, assurent mal les évacuations. Mais l'agriculture moderne n'est pas en reste, et s'active à produire de plus en plus de nuisances chimiques, alors que l'industrie n'occupe au mieux qu'une moitié du quart des disponibilités en eau, l'autre moitié allant aux besoins urbains, et les trois quarts du total au moins revenant évidemment à l'agriculture. De plus, il est infiniment plus onéreux en ressources naturelles, eau et sols, de réaliser une plus-value en agriculture, et avec un toit de saturation plus vite atteint, pendant que pour l'industrie, les possibilités d'amélioration et d'adaptation semblent infinies. Certains pays de la région sont entrés dans un processus d'industrialisation propre, et seraient capables de reprendre la noble action de «fabrication industrieuse», en faisant une pratique assez saine et durable, à l'orée de ce qui serait leur révolution industrielle; celle qu'ils n'ont jamais faite, et que le Monde actuel appelle de ses vœux, sous le nouveau nom de « transition écologique ». Enfin, tout point gagné par ces pays oasiens, en matière de développement sur une base plus nettement industrielle, serait assurément aussi un cran positif en matière de promotion scientifique et technique, et éventuellement d'égard pour une puissance naissante.

Faut-il aller jusqu'à penser que s'il devait y avoir des cycles universels de développement, on serait maintenant à méditer des possibilités de renaissance des ensembles oasiens? Les oasis d'Asie centrale seraient stimulées en proportion des centres de polarité économique extrême-orientaux, pendant que l'Afrique, qui promet des lendemains qu'on voudrait meilleurs, a besoin de se réapproprier son Sahara, par les meilleures voies de pénétration possibles.

Enfin, on est en droit d'attendre des grandes puissances une «sainte alliance environnementale», à même de réparer certains des torts, infligés aux pays du tiers monde, du fait de comportements trop systématiquement extractifs, et de transferts massifs des ressources naturelles. On pourrait même dire que les cadres institutionnels pour le développement territorial durable, et qui sont transnationaux pour certains, existent déjà, même s'ils n'ont, au mieux, qu'un caractère expérimental. Ils pourraient proposer, notamment, des formules de terrain, telles que celles lancées par l'Unesco, du type «réserves de biosphère» ou «sites du patrimoine mondial», sur les traces de «la Route de la soie» en Asie, ou à la recherche des empires africains plus ou moins «dorés», en croisant des repères prestigieux tels que le 


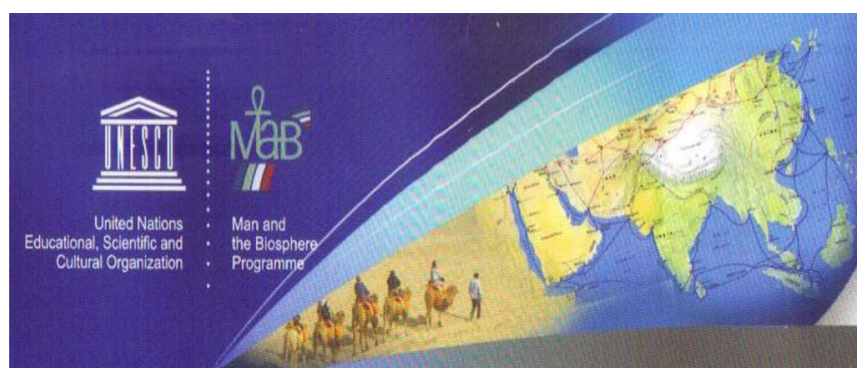

Fig. 13. L'espace oasien central, depuis la fracture qui mutile le monde vivant, jusqu'au trait d'union de tous les espoirs.

Fig. 13. The oasis areas: a great divide in the living world but also a link to hope.

Tafilalt ou Tamenghest (Fig. 13). Plus qu'on ne saurait l'imaginer, ces formules, déjà organisées en réseaux, pourraient faciliter les échanges d'expériences et normaliser le développement durable à l'échelle des grandes régions du Globe (Unesco, 2017).

\section{Références}

Barnes FA, Pendleton M. 1979. Canyon country; prehistoric Indians, their cultures, ruins, artifacts and rock art. Salt Lake City, Utah: Wasatch Publishers Inc., 256 p.
Bouaziz A. 2000. L'agrodiversité dans la réserve de biosphère des Oasis du Sud marocain. In : Dossier de candidature de la RBOSM. Paris: Unesco, Divis. Sc. Ecolo. Terre.

Fassi D. 1999. La forêt dans l'histoire de la Terre. In : Le grand livre de la forêt marocaine. Belgique: Mardaga, $280 \mathrm{p}$.

Fassi D. 2001. Contraintes et potentialités de l'agriculture maghrébine. Présentation des milieux physique et humain. $C R$ Acad Agric Fr 87 (2): 129-145.

Guilaine J. 2000. Premiers paysans du monde. Naissances des agricultures. Séminaire du Collège de France. Collection des Hespérides. Paris: Éditions errance, 320 p.

Ibn Khaldoun A. 1377. Mouqaddima. (Prolégomènes). Présentée par Abou Abdarrahman Adel Ibn Saad, Addar Addahabia, Le Caire, 2006, 735 p. (en arabe). Traduction en français : http://classiques. uqac.ca/classiques/Ibn_Khaldoun/Prolegomenes_t1/Prolegome nes t1.html.

Lahmar R. 1997. L'opération terres vierges du Kazakhstan. Alliance pour un monde responsable. Gestion durable des sols. Disponible sur: http://www.alliance21.org/2003/article931.html.

Miller JA. (sans date). Environmental organization in the Tafilalt Oasis: ancient Sijilmassa and contemporary development. Clemson University et Centre pour études alaouites à Rissani: Maroc.

Unesco. 2000. Réserve de biosphère des Oasis du Sud marocain (RBOSM). Dossier de candidature de la RBOSM. Paris: Unesco, Divis. Sc. Ecolo. Terre. Supervisé par Fassi D.

Unesco. 2017. A new roadmap for the Man and the biosphere (MAB) programme and its world network of biosphere reserves. Paris: Man and the Biosphere Programme; United Nations Educational, Scientific and Cultural Organization, $55 \mathrm{p}$.

Citation de l'article : Fassi D. 2017. Les oasis du Monde, carrefour des civilisations et modèle fondamental de durabilité. Cah. Agric. 26: 46001 . 